\title{
Transcription termination at the Escherichia coli thra terminator by spinach chloroplast RNA polymerase in vitro is influenced by downstream DNA sequences
}

\author{
Liang-Jwu Chen ${ }^{\star}$, Yuh-Jin Liang, Shih-Tong Jeng ${ }^{1}$, Emil M. Orozco², \\ Richard I. Gumport ${ }^{3}$, Chi-Hui Lin and Ming-Te Yang
}

\begin{abstract}
Institute of Molecular Biology, National Chung Hsing University, Taichung 40227, Taiwan, Republic of China, 1Department of Botany, National Taiwan University, Taipei, Taiwan, Republic of China, ${ }^{2}$ DEKALB Plant Genetics, 62 Maritime Drive, Mystic, CT 06355, USA and ${ }^{3}$ Department of Biochemistry, College of Medicine and School of Chemical Sciences, University of Illinois, Urbana, IL 61801, USA
\end{abstract}

Received July 24, 1995; Revised and Accepted October 20, 1995

\begin{abstract}
We have investigated the mechanism of transcription termination in vitro by spinach chloroplast RNA polymerase using templates encoding variants of the transcription-termination structure (attenuator) of the regulatory region of the threonine (thr) operon of Escherichia coli. Fourteen sequence variants located within its $d(G+C)$ stem-loop and $d(A+T)$-rich regions were studied. We found that the helix integrity in the stem-loop structure is necessary for termination but that its stability is not directly correlated with termination efficiency. The sequence of the $G+C$ stem-loop itself also influences termination. Moreover, the dA template stretch at the $3^{\prime}$ end of the terminator plays a major role in termination efficiency, but base pairing between the $A$ and $U$ tract of the transcript does not. From the studies using deletion variants and a series of mutants that alter the sequences immediately downstream from the transcription termination site, we found that termination of transcription by spinach chloroplast RNA polymerase was also modulated by downstream DNA sequences in a sequence-specific manner. The second base immediately following the poly( $T)$ tract is crucial for determining the termination efficiency by chloroplast RNA polymerase, but not of the $T 7$ or E.coli enzymes.
\end{abstract}

\section{INTRODUCTION}

Most plastid genes contain inverted repeat sequences at their $3^{\prime}$ ends that are thought to confer upon their transcripts the ability to form stem-loop structures. Such structures were initially presumed to be transcription terminators, analogous to bacterial factor-independent terminators. However, when they were analyzed in a homologous in vitro transcription system, no significant termination activity was observed $(1,2)$. It was subsequently concluded that the $3^{\prime}$ ends of the $r b c L, a t p B$, psbA, petD and $r p o A$ transcripts from spinach chloroplast are primarily the result of RNA processing and not transcription termination $(2,3)$. In contrast, a recent report indicates that the stem-loop structure of the $3^{\prime}$ ends of the Chlamydomonas chloroplast $r b c L$ and psaB genes appear to have significant termination activity in vivo (4). In addition, the prokaryotic factor-independent terminators that contain a typical $\mathrm{d}(\mathrm{G}+\mathrm{C})$-rich region of dyad symmetry and are followed by a poly(T) tract in the sense strand, such as the terminator for the threonine attenuator, thra from Escherichia coli, efficiently terminate transcription in vitro by spinach chloroplast RNA polymerase $(1,5)$. This indicates that the transcription termination process in chloroplasts may have at least some features in common with the mechanism used in prokaryotes and that the stem-loop-dependent termination is probably sometimes functional in chloroplasts.

The mechanism of transcription termination for E.coli factorindependent terminators has been well characterized. A classical thermodynamic model $(6,7)$ for factor-independent terminators proposes that termination occurs after the $\mathrm{G}+\mathrm{C}$-rich region forms a stable base-paired stem-loop structure in the RNA. This causes the polymerase to pause and release the elongating transcript. Release may also be enhanced by the instability of the dA-rU base pairs that often comprise the remainder of the transcript/template hybrid $(8,9)$. Many studies involving base analogues $(10,11)$ heteroduplexes (12) and mutant terminators for E.coli thra terminator (13-16) support this model. Transcription termination by the E.coli thra terminator with T7 RNA polymerase indicates that the dyad symmetry, the poly $(\mathrm{T})$ tract and many other sequence elements also affect termination $(13,14)$. However, studies indicating that the sequences downstream from the termination site affect termination efficiency $(17,18)$ can not be explained by a simple thermodynamic model. Recent findings about the cleavage of the nascent RNA in the transcriptional complex (19-21), the existence of RNA binding sites on RNA polymerase (19) and the observation of discontinuous movements of DNA and RNA with respect to RNA polymerase during elongation (22-25) and pausing (26) led to an altemative model of transcription termination (27). This inchworming model describes transcription termination as coupling pulsate RNA polymerase movement, and the poly(T) tract that follows the stem-loop structure acts as an inchworming signal (27).

Since the E.coli thra terminator is functionally recognized by spinach chloroplast RNA polymerase, the study of the thra terminator variants in a transcription system using chloroplast 
RNA polymerase should help us further understand transcription termination in chloroplasts. In this work, we studied the effect of a series of mutations in the thra terminator on transcription termination with spinach chloroplast RNA polymerase. Included in this study were 14 mutants with single base substitutions in the $\mathrm{d}(\mathrm{G}+\mathrm{C})$-rich and $\mathrm{d}(\mathrm{A}+\mathrm{T})$-rich regions and seven nested deletion variants in the poly $(T)$ tract region. Many other variants in the downstream region of the thra terminator were also used. The

A) pTZ19-Pus TreT.

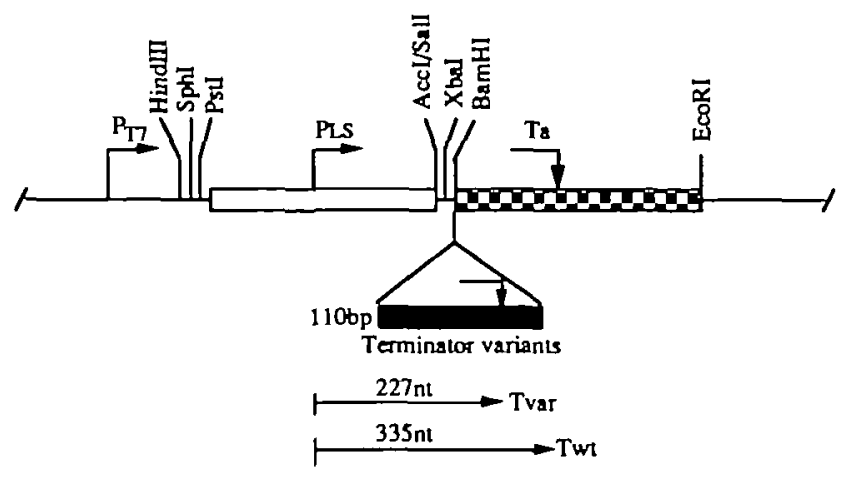

B) thra terminator RNA stem-loop

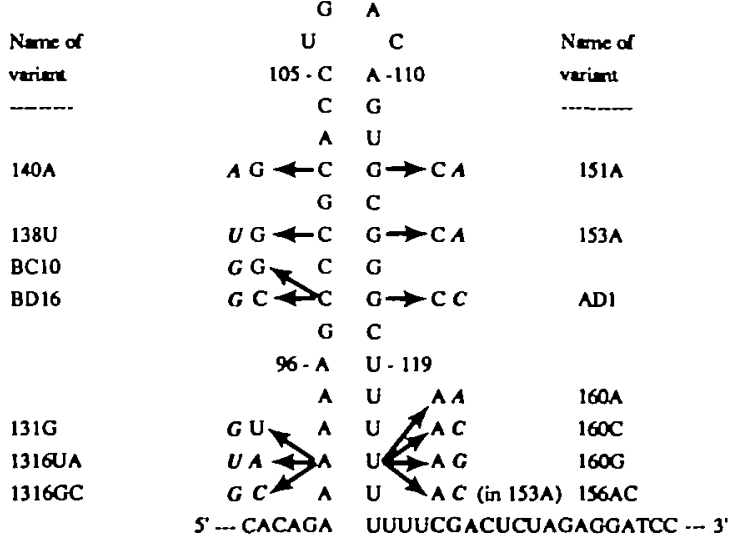

C) Sequences of the $110 \mathrm{bp}$ thra terminator and lts variants GGATCCTCAACTGTGAGGAGGCTCACGGACGAAGAACAGGCACGCGTACAGGAAA BumHI
CACAGAAAAAAGCCCGCACCTGACAGTGCGGOCTTTTTTTTCGACTCTAGAGGATLC \begin{tabular}{ll} 
TS TTTTTTT- CTAGAGGATCC \\
T6 TTTTT - CTAGAGGATCC \\
TS TTTTT - CTAGAGGATCC \\
T4 \\
TTTT \\
TS TTT -CTAGAGGATCC \\
T2 & TT \\
Ti & T -CTAGAGGATCC \\
\hline
\end{tabular}

D) Sequences of the thre terminator and T8WT, T8 $\triangle$ and YNANN vartants GGATCCTCAACTGTGAGGAGGCTCACGGACGAAGAACAGGCACGCGTACAGGAAA CACAGAAAAAAGCCCGCACCTGACAGTGCGGGCTTTTTTTTTCGACTCTAGAGGATCC

\begin{tabular}{|c|c|}
\hline TOHT(TECGACT) & TTTTTTTT-COACTCTAGA \\
\hline YNANN vartends & TTTTTTTT $\sqrt[\text { YNANA FTAGA }]{ }$ \\
\hline$T 8 \triangle(T \& \triangle C I \wedge O A)$ & TTITITTT-CTAOAGGATCC \\
\hline T\&CGAGA & TTTTTTTP____C CGAGAGGATCC \\
\hline 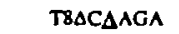 & TTTTTTT-_- CANOAGGATCC \\
\hline TBACCAGA & TTTTTTT-...... CQAGAGGATCC \\
\hline
\end{tabular}

results indicate that the specific sequence as well as the helix stability within the $d(\mathrm{G}+\mathrm{C})$-rich region, and the length of the poly(T) tract affect termination efficiency. In addition, we made the unexpected observation that untranscribed sequence(s) downstream of the thra terminator play a significant role in termination efficiency with spinach chloroplast RNA polymerase.

\section{MATERIALS AND METHODS}

\section{Plasmid DNA constructions}

The plasmid pTZ19-PLSTa contains the 5'-end of the spinach $r b c L$ gene promoter (PLS) and the terminator from the threonine attenuator region (Ta) as previously described (1). The terminator variants were originally from the pTZ-19thr variants and pTZ-19T series as described by Jeng et al. (13). The 110 bp BamHI DNA fragments containing the various terminator mutants were purified and ligated to the BamHI site of plasmid pTZ19-PLSTa. The orientation of the BamHI DNA fragments in each clone was confirmed. For all of the nested deletion variants, the sequence immediately following the template deoxyadenosine tract had the 5 deoxynucleotides CGACT deleted. In these nested deletion variants, the variant contains an $8 \mathrm{~T}$ tract was

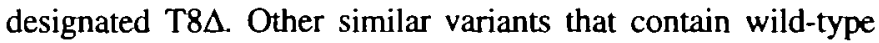
downstream sequences were designated T8WT. A schematic diagram for the construction of terminator is presented in Figure 1 and their characteristics are summarized in Table 1.

Besides the terminator variants that have sequences changed in $d(G+C)$-rich hairpin and $d(A+T)$-rich regions, a series of mutants that have sequences changed downstream of the termination site were constructed. These downstream sequence mutants were created by PCR with a degenerate primer YNANN (Fig. ID, Y $=\mathrm{Cor} T, \mathrm{~N}=\mathrm{G}, \mathrm{A}, \mathrm{C}$ or $\mathrm{T}$ ) and a reverse primer, the variant T8WT was used as template for DNA fragment amplification. The YNANN primer will theoretically create 128 mutants. The $110 \mathrm{bp}$ DNA fragments that may contain all the 128 different downstream sequence combinations were cut out from the PCR amplified products with $\mathrm{BamHI}$ and then cloned into the BamHI site of PTZ-PLSTa. The different downstream sequence combinations in each isolated clone was identified by sequencing. The other set of mutants T8 $\triangle$ CGAGA, T8 $\triangle$ CCAGA and T8 $\triangle$ CAAGA, were also constructed by PCR with a primer that . has the nucleotides $\mathrm{G}, \mathrm{A}$ or $\mathrm{C}$ mixed at the position indicated; the variant T8 (also designated as T8

Figure 1. Schematic diagrams of plasmid DNAs pTZ19-PLSTvarTa and structure of thra terminator RNA stem-loop and its variants. (A) The terminator variants were constructed by inserting the various 110 bp BamHI DNA fragments from pTZ-19T series, pTZ-19thr variants (13) and YNANN mutants into the BamHI site of plasmid PTZ19-PLSTa (1). The sizes for transcripts initiating at the 'PLS' promoter and terminated at the terminator variants (227 $\mathrm{nt}$ ) or at the wild-type terminator ( $335 \mathrm{nt}$ ) were indicated. (B) The secondary structure of the wild-type thra terminator is presented as the conformatuon that maximizes potential base pairing. The RNA numbering system is same as in Table 2 and the nucleotide positions are relative to the transcription initiation site of 77 RNA polymerase as described previously (13). The changed bases in each variant are indicated as italics and shown in Table 2 . (C) The nested deletion variants with shortened d(T) tract are shown. Five nucleotides located downstream from termination release sites were deleted as indicated by dash line. (D) The relative location of sequences changed for YNANN mutants is shown. A degenerate primer contain mixed nucleotides are indicated by the letter $\mathrm{Y}=\mathrm{C}$ or $\mathrm{T}$ and $\mathrm{N}=\mathrm{G}, \mathrm{A}, \mathrm{T}$ or $\mathrm{C}$ and the variant T8WT was used as DNA template. The single base change for terminator variants T8 $\triangle \mathrm{CGAGA}$,

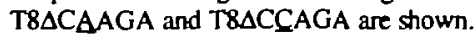


Table 1. Vectors and the thra terminator variants used in this study

\begin{tabular}{|c|c|c|}
\hline Vectors or variants & Characteristics & Reference or source \\
\hline TZ19T series & Contains the nested deletion variants & $(13)$ \\
\hline pTZ19 thr variants & Contains the 14 variants in $G+C$ rich and $A+T$ rich regions & (13) \\
\hline pTZ19PLSTa & Contains a chloroplast promoter and the wild-type thra termunator & (1) \\
\hline pTZ19PLSTvarTa & $\begin{array}{l}\text { All thra terminator variants in this study are cloned between the } \\
\text { chloroplast promoter and the wild-type thra terminator }\end{array}$ & This work \\
\hline YNANN variants & $\begin{array}{l}\text { Contains the thra terminator vanants with various downstream } \\
\text { sequences combinations }\end{array}$ & This work \\
\hline T8DCNAGA vanants & $\begin{array}{l}\text { Contains four variants that each has only one base changed at the } \\
\text { position indicated }\end{array}$ & This work \\
\hline
\end{tabular}

\section{Analysis of in vitro transcripts}

Transcriptionally active extracts of spinach chloroplasts were prepared as previously described (1). Each standard in vitro transcription reaction $(40 \mu \mathrm{l})$ contained $12 \mathrm{mM}$ Hepes- $\mathrm{KOH}$ (pH 8), $40 \mathrm{mM} \mathrm{KCl}, 10 \mathrm{mM} \mathrm{MgCl} 2,10 \mathrm{mM} \mathrm{DTT}, 1 \mathrm{U} / \mu \mathrm{l}$ RNase inhibitor, $500 \mu \mathrm{M}$ ATP, UTP and CTP and $10 \mu \mathrm{M}$ GTP containing $10 \mu \mathrm{Ci}\left[\alpha-{ }^{32} \mathrm{P}\right] \mathrm{GTP}(800 \mathrm{Ci} / \mathrm{mmol}, 10 \mu \mathrm{Ci} / \mu \mathrm{l} ; \mathrm{NEN}$, Dupont $)$ and the following components: one-tenth of the volume $(4 \mu \mathrm{l})$ was supercoiled or linearized DNA $(0.4 \mu \mathrm{g})$ in $10 \mathrm{mM}$ Tris- $\mathrm{HCl}$ $(\mathrm{pH} 8), 0.1 \mathrm{mM}$ EDTA and two-fifths of the volume $(16 \mu \mathrm{l})$ was chloroplast RNA polymerase high-salt extract in DEAE buffer [ $50 \mathrm{mM}$ Tricine-KOH (pH 8), $50 \mathrm{mM} \mathrm{KCl}, 0.5 \mathrm{mM}$ EDTA, $0.5 \mathrm{mM}$ DTT, $0.5 \mathrm{mM}$ phenylmethylsulfonyl fluoride, $1 \mathrm{mM}$ benzamidine, $5 \mathrm{mM} \varepsilon$-amino-n-caproic acid, $5 \%(\mathrm{v} / \mathrm{v})$ glycerol] or $12.5 \mathrm{U}$ of T7 RNA polymerase (in DEAE buffer). The DEAE buffer contributed an additional $20 \mathrm{mM}$ of $\mathrm{KCl}$ to the final reaction volume. The in vitro transcription reaction was incubated at $30^{\circ} \mathrm{C}$ (for chloroplast RNA polymerase) or at $37^{\circ} \mathrm{C}$ (for $\mathrm{T} 7$ RNA polymerases) for $60 \mathrm{~min}$. The reaction was then stopped by the addition of $40 \mu$ l of RNA extraction buffer [ $6 \mathrm{M}$ urea, $0.36 \mathrm{M}$ $\mathrm{NaCl}, 20 \mathrm{mM}$ EDTA, $10 \mathrm{mM}$ Tris- $\mathrm{HCl}(\mathrm{pH} 8)$ and 1\% SDS]. E.coli tRNA ( $15 \mu \mathrm{g} /$ reaction) was added as carrier and the in vitro RNA extracted twice with phenol:chloroform and ethanol-precipitated. The nucleic acid pellet was rinsed once with cold $80 \%$ ethanol, dried and resuspended in $80 \%$ formamide, $0.1 \%$ bromophenol blue and $0.1 \%$ xylene cyanol. The in vitro transcription products were analyzed by electrophoresis through $8 \%$ polyacrylamide-8.3 $\mathrm{M}$ urea DNA sequencing gels and subsequent autoradiography.

\section{Quantification of termination efficiency}

The autoradiograms were scanned with an LKB Ultroscan XL Laser Densitometer or the ${ }^{32} \mathrm{P}$ in RNA-containing gel slices was measured by liquid scintillation counting. Densitometry of multiple autoradiographic film exposures was performed to ensure that the autoradiographic signal was within the linear response range of the film. Each dataset was corrected for background and normalized according to the length and guanosine composition of each transcript. The data from multiple experiments were expressed as the average value \pm one standard error. The number of repetitions for each experiment is indicated in the tables. The termination efficiency for each variant was calculated essentially as described by Chen (28). After the absolute efficiency of termination for each variant was obtained, the termination efficiency for each variant relative to the wild-type was calculated (Table 2).

\section{RESULTS}

\section{Transcription in vitro with thra terminator variants by spinach chloroplast RNA polymerase}

We used 21 thra terminator variants, including point substitutions in the $d(G+C)$ rich region of dyad symmetry, point substitutions in the $d(A+T)$ rich region (Fig. 1B) and a set of nested deletions in the poly(T) tract (Fig. 1C) of the thra terminator to study transcription termination by spinach chloroplast RNA polymerase. Each thra terminator variant, consisting of a $110 \mathrm{bp} \mathrm{BamHI}$ DNA fragment, was cloned into the BamHI site of plasmid pTZ19-PLSTa to get the plasmids designated pTZ19-PLSTvarTa (Fig. 1A). Thus, each construct contained two terminator sequences: (i) an altered thra terminator located at the BamHI site, and (ii) immediately downstream, a wild-type thra terminator (from the plasmid vector pTZ19-PLSTa) to serve as an internal control (Fig. 1A). The CsCl-prepared supercoiled plasmid DNAs were used as DNA templates. Transcription reactions and their products were analyzed as described in Materials and Methods and the results are shown in Figure 2. All thra terminator variants, no matter where the mutation was located, decreased the amount of termination when compared with the wild-type terminator (Fig. 2, lanes 2 and 3-16). Their relative efficiencies of transcription termination by spinach chloroplast RNA polymerase and T7 RNA polymerase are presented in Table 2.

The wild-type thra-terminated RNAs (Twt) synthesized from templates encoding the terminator variants in the $d(A+T)$-rich region $(131 \mathrm{G}, 1316 \mathrm{UA}, 160 \mathrm{~A}, 160 \mathrm{C}$ and $160 \mathrm{G})$ and variant $\mathrm{BD} 16$, which does not create a mismatch in the $\mathrm{G}+\mathrm{C}$ region of the transcript, migrated at the same rate as the transcript formed by the wild-type template (Fig. 2, lane 2 versus lanes 3-8). The RNAs synthesized from variant $1316 \mathrm{GC}$, which created one potential GC base pair in the A+T-rich region and accordingly increased the calculated helix stability, migrated more slowly than the wild-type transcripts (Fig. 2, lane 9 versus lanes 2-8). Conversely, the RNAs synthesized from the terminator variants that caused mismatches in the $\mathrm{G}+\mathrm{C}$-rich hairpin region migrated more rapidly than the transcript formed by wild-type template (Fig. 2, lanes 10-16). These mobility anomalies have been attributed to differences in stability of the RNA helices $(13,16)$. 
Table 2. Relative termination efficiencies of thra terminator variants

\begin{tabular}{|c|c|c|c|c|c|}
\hline Template & $\begin{array}{l}\text { Positions } \\
\text { altered }^{\mathbf{a}}\end{array}$ & $\begin{array}{l}\text { Base pair } \\
\text { change }\end{array}$ & $\begin{array}{l}\Delta \mathrm{G} \\
\mathrm{kcal} / \mathrm{mol}^{\mathrm{b}}\end{array}$ & $\begin{array}{l}\text { Spinach } \\
q_{0} \mathrm{c}\end{array}$ & $\begin{array}{l}T 7 \\
90 d\end{array}$ \\
\hline wild-type & & & -23 & $100^{x}$ & $100^{f}$ \\
\hline $131 \mathrm{G}$ & 93 & $\mathrm{AU} \rightarrow \mathrm{GU}$ & -22 & $77 \pm 5$ & 100 \\
\hline $153 \mathrm{~A}$ & 115 & $\mathrm{CG} \rightarrow \mathrm{CA}$ & -16 & $48 \pm 5$ & 30 \\
\hline $151 \mathrm{~A}$ & 113 & $\mathrm{CG} \rightarrow \mathrm{CA}$ & -17 & $43 \pm 2$ & 16 \\
\hline BDI6 & $98 ; 117$ & $\mathrm{CG} \rightarrow \mathrm{GC}$ & -23 & $29 \pm 3$ & 42 \\
\hline $138 \mathrm{U}$ & 100 & $\mathrm{CG} \rightarrow \mathrm{UG}$ & -21 & $28 \pm 2$ & 33 \\
\hline $140 \mathrm{~A}$ & 102 & $\mathrm{CG} \rightarrow \mathrm{AG}$ & -17 & $26 \pm 3$ & 14 \\
\hline $1316 \mathrm{UA}$ & $93 ; 122$ & $\mathrm{AU} \rightarrow \mathrm{UA}$ & -23 & $20 \pm 4$ & 44 \\
\hline $160 \mathrm{~A}$ & 122 & $\mathrm{AU} \rightarrow \mathrm{AA}$ & -21 & $18 \pm 2$ & 47 \\
\hline $1316 \mathrm{GC}$ & $93 ; 122$ & $\mathrm{AU} \rightarrow \mathrm{GC}$ & -25 & $15 \pm 3$ & 30 \\
\hline $160 \mathrm{C}$ & 122 & $\mathrm{AU} \rightarrow \mathrm{AC}$ & -21 & $13 \pm 3$ & 31 \\
\hline $160 \mathrm{G}$ & 122 & $\mathrm{AU} \rightarrow \mathrm{AG}$ & -21 & $12 \pm 3$ & 30 \\
\hline ADI & 117 & $\mathrm{CG} \rightarrow \mathrm{CC}$ & -16 & $10 \pm 4$ & 9 \\
\hline $\mathrm{BC} 10$ & 98 & $\mathrm{CG} \rightarrow \mathrm{GG}$ & -16 & $<10$ & 12 \\
\hline \multirow[t]{2}{*}{$156 \mathrm{AC}$} & 115 & $\mathrm{CG} \rightarrow \mathrm{CA}$ & & & \\
\hline & 122 & $\mathrm{AU} \rightarrow \mathrm{AC}$ & -14 & $<10$ & 9 \\
\hline
\end{tabular}

The positions correspond to those shown in Figure 1.

${ }^{b} \Delta G$ values were calculated according to Freier et al. (29).

'Transcription termınation was calculated as described in the Materials and methods. Each data represents the average of three individual experiments.

tThese transcription termination data were derived from Jeng et al. (13).

The absolute termination efficiency is $63 \%$ (28).

fThe absolute termination efficiency is $43 \%$ according to Jeng et al. (13).

Our results confirm that calculated stability was well correlated with relative mobility of the terminator variant transcripts (Fig. 2 and Table 2). The anomalous migrations we observed were obtained for the transcripts produced by terminating at the Twt. The transcripts that terminated at the variant terminators (Tvar) migrated normally. In contrast, others have reported that the anomalous migrations were observed in the smaller transcripts due to the terminator variants, while the longer run-off transcripts migrated normally $(13,16)$. The presence of tandem hairpins, due to the two terminators, may contribute to the different conformations observed in this study.

\section{Termination with variants at the $\mathrm{d}(\mathrm{G}+\mathrm{C})$-rich hairpin region}

Seven variants $(138 \mathrm{U}, 153 \mathrm{~A}, 140 \mathrm{~A}, 151 \mathrm{~A}, \mathrm{BD} 16, \mathrm{BC} 10$ and $A D 1)$ in the $d(G+C)$-rich region were studied. The efficiency of termination from these variants ranged from $<10$ to $48 \%$ of the wild-type terminator. Among these variants, BD16, BC10 and ADl existed in a slightly different sequence context than the remainder of the constructions. In these three variants the template poly(T) tract was shortened from 9 nucleotides (nt), as it in the wild-type thra terminator, to $8 \mathrm{nt}$. The termination efficiencies of two of these variants, BCI0 $(<10 \%)$ and AD1 $(10 \%)$, were substantially less than those of other mismatch variants 140A (26\%), 153A (48\%) and 151A (43\%). It is plausible that the decreased termination efficiencies in these variants were due to both the mismatches in $\mathrm{d}(\mathrm{G}+\mathrm{C})$-rich region and a one-T shortening of poly( $T)$ tract length. However, efficiencies of termination by both E.coli and T7 RNA polymerase $(13,16)$ and spinach chloroplast RNA polymerase (Fig. 3, lanes T9WT versus T8WT, and Table 3) were unaffected by changes in poly(T) template from 9-T to 8-T. Hence, the low termination efficiencies for $\mathrm{BC} 10$ and $\mathrm{ADl}$ variants are more likely due to the changes in the $d(G+C)$-rich hairpin and are affected only slightly by the length of the poly(T) tract. The mismatch variants (140A, 153A, 151 A, BCl0 and AD1) destablize the helix and decrease termination, suggesting that helix integrity in the stem of the stem-loop structure is necessary for efficient termination.

Table 3. The termination efficiency of the thra terminator variants uanscribed with chloroplast or T7 RNA polymerase

\begin{tabular}{|c|c|c|c|}
\hline \multirow[t]{2}{*}{ Variants } & \multirow{2}{*}{$\begin{array}{l}\text { Composition of } \\
\text { downstream sequences }\end{array}$} & \multicolumn{2}{|c|}{ Termination efficiency $\boldsymbol{q}^{\mathrm{a}}$} \\
\hline & & Chloroplast & $\mathrm{T7}$ \\
\hline T9WT & T9CGACT & $62.6 \pm 4.0$ & $64.4 \pm 2.4$ \\
\hline T8WT & T8CGACT & $61.8 \pm 4.5$ & $63.3 \pm 1.6$ \\
\hline T8DCTAGA & T8CTAGA & $9.2 \pm 1.1$ & $63.2 \pm 1.9$ \\
\hline T8DCGAGA & T8CGAGA & $54.4 \pm 3.7$ & $65.1 \pm 1.6$ \\
\hline T8DCAAGA & T8CAAGA & $8.4 \pm 1.3$ & $62.3 \pm 0.8$ \\
\hline T8DCCAGA & T8CCAGA & $11.7 \pm 2.9$ & $58.9 \pm 2.0$ \\
\hline
\end{tabular}

Transcription termination was calculated as described in the Materials and Methods. Each data represents the average of three individual experiments.

To study the relationship between the termination efficiency and helix integrity, stability values were calculated by the method of Freier et al. (29). These calculations indicated that although the variants $138 \mathrm{U}$ (stability $=-21 \mathrm{kcal} / \mathrm{mol})$ and $140 \mathrm{~A}(-17 \mathrm{kcal} / \mathrm{mol})$ 


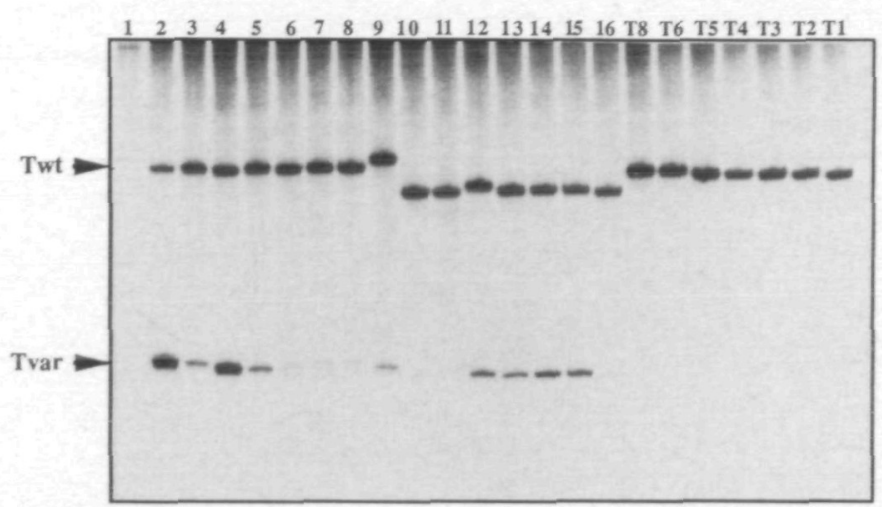

Figure 2. In vitro transcription of the thra stem-loop terminator variants and the poly $(\mathrm{T})$ tract shortened variants with chloroplast RNA polymerase. The transcripts were prepared and analyzed as described in Materials and Methods. The transcripts terminated at the terminator variants (Tvar) and at the wild-type terminator (Twt) are indicated. Lane 1, no DNA; lane 2, wild-type (T9WT) terminator located at the Tvar site; lane 3, 1316UA; lane 4, 131G; lane 5, BD16; lane 6, 160A; lane 7, 160C; lane 8, 160G; lane 9, 1316GC; lane 10, 156AC; lane 11, BC10; lane 12, 138U; lane 13, 140A; lane 14, 151A; lane 15, 153A; lane 16, AD1; lanes T8, T6, T5, T4, T3, T2, T1 are 8, 6, 5, 4, 3, 2, 1 deoxyadenosine variants, respectively.

are thermodynamically stable, they have low termination efficiencies, whereas the variants $151 \mathrm{~A}(-17 \mathrm{kcal} / \mathrm{mol})$ and $153 \mathrm{~A}$ $(-16 \mathrm{kcal} / \mathrm{mol})$ with similar or lower stability, have much higher termination efficiency (Table 2). These finding indicated that there is no direct correlation between the decrease in helix stability and the decrease in termination efficiency. In addition, the variants $138 \mathrm{U}$ and $140 \mathrm{~A}$, which have their sequences altered at positions \#100 and \#102, respectively, had lower termination efficiencies than the variants $151 \mathrm{~A}$ and $153 \mathrm{~A}$ with the same mismatch base pairing but with their sequences altered at positions \#113 and \#115 on the opposite side of the stem structure. This finding indicated that not only the mismatch but the sequence itself influenced termination. Consistent with this interpretation, the variant BD16, that forms an inverted GC base pair and has the same calculated stability value $(-23 \mathrm{kcal} / \mathrm{mol})$ as the wild-type, had a termination efficiency of only $29 \%$ of the wild-type value.

\section{Termination with variants at the $d(A+T)$-rich region}

Seven variants that have sequences altered in both the runs of (dA) and (T) residues were used to determine the effects of changes in the sequence of the $\mathrm{d}(\mathrm{A}+\mathrm{T})$ rich region of the thra terminator (Fig. 1B). Three variants $(160 \mathrm{~A}, 160 \mathrm{C}$ or $160 \mathrm{G})$, which disrupted the $\mathrm{T}$ tract ( $\mathrm{U}$ tract in transcript) by substituting $A, C$ or $G$ for $U$ at position 122 , decreased termination to $\leq 18 \%$ of the wild-type value (Table 2). Two variants, 1316UA and 1316GC, which change both the $A$ and $U$ stretches at positions 93 and 122 , respectively, allow base pairing at this site; they nevertheless decrease termination efficiencies. The termination efficiency of $1316 \mathrm{UA}$ (20\%) is nearly the same as that of $160 \mathrm{~A}$ $(18 \%)$, which contains a substitution of $A$ with $U$ at position 122 that creates a mismatch. Similarly, variant $160 \mathrm{C}$, which disrupts the poly-U tract and creates a mismatch, terminates with the same efficiency as does the variant $1316 \mathrm{GC}$. In addition, the variant $131 G$ that changes $A$ to $G$ at position 93 in the poly-A tract has

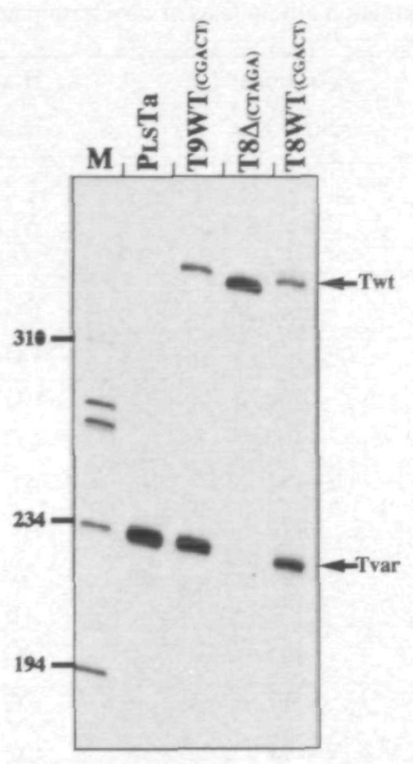

Figure 3. In vitro transcription of the thra terminator variants T9WT, T8 $\Delta$ and T8WT with chloroplast RNA polymerase. Lane M: $\phi$ X174-HaeIII molecular size standards. The lengths of denatured DNA size standards are shown as well as the positions of the transcripts that terminated at the terminator variants (Tvar) and at the wild-type terminator (Twt). The transcript in lane PLSTa, which contain only a wild-type terminator as described in Chen and Orozco (1), is the transcript that terminates at the wild-type terminator.

a minor effect on termination. These results indicate that the poly-U stretch plays a major role in termination efficiency and that the base pairing between the $A$ and $U$ tract of the transcript does not play a crucial role in termination.

Another variant (156AC), which contains two AC mismatches, one in the $\mathrm{G}+\mathrm{C}$-rich region (as in 153A) and one in the $\mathrm{A}+\mathrm{T}$-rich region (as in $160 \mathrm{C}$ ), decreases termination to barely detectable levels suggesting that the two base changes may independently contribute to disrupting termination.

\section{Effects of the length of $T$ tract and of the variants T8 $\Delta$ and T8WT}

Lynn et al. (16) reported that the length of the deoxyadenosine tract of the template, encoding the stretch of uridines at the $3^{\prime}$ end of the transcript, affects the termination efficiency with E.coli RNA polymerase. A series of nested deletion variants that successively shorten the deoxyadenosine tract in the terminator template (13 and Fig. 1C) were used to test their function with chloroplast RNA polymerase. The nested deletion variants exist in a slightly different sequence context than does the wild-type construction. In all of the nested deletion variants, the sequence of 5 deoxynucleotides that immediately following the template deoxyadenosine tract in the wild-type, $\mathrm{d}(\mathrm{CGACT})$, were deleted as a result of the cloning procedures (13). In this study, we have cloned these nested deletion variants of the thra terminator in a series of plasmids that also contains the wild-type thra terminator immediately downstream as the internal control (Fig. 1A). The results of successively shortening the run of deoxyadenosines in the terminator template are shown in Figure 2 (lanes T8-T1). There was only a background level of termination at Tvar for variant $\mathrm{T} 8$, and no detectable signal for any of the other shortened 
T variants, while the wild-type thra terminator at Twt terminated transcription normally (Fig. 2, bands Twt). Although these data might be explained by extreme sensitivity of the chloroplast enzyme to a shortened $T$ tract, the variant BD16, which also contained a shortened 8-deoxyadenosine tract, had a termination efficiency of $\sim 29 \%$ of the wild-type value. BD16 differed from the deletion mutants, however, in that its sequence downstream of the poly-T was intact. Taken together, the loss of termination function in these nested deletion variants with chloroplast RNA polymerase could be attributed to the combination of changes in the 5 deoxynucleotides immediately downstream from the transcription termination site as well as the shortened $T$ tract.

To test how the downstream sequences affect the termination efficiency, a variant T8WT, which contain the wild-type downstream sequences and 8 deoxyadenosine tract, was used along with a T8 variant, one of the nested deletion variants which contained 8 deoxyadenosine tract and has 5 deoxynucleotides CGACT deleted (we have renamed this variant T8 $\Delta$ for easy comparison; Fig. 1D). These two variants contain an identical structure in the $d(\mathrm{G}+\mathrm{C})$-rich region and the $T$ tract. The only difference between them is that in T8 $\Delta$ the sequence CGACT has been deleted resulting in the $\mathrm{T} 8$ tract being adjacent to the sequence CTAGA (Fig. 1D). T8WT had about the same efficiency of termination as T9WT (Fig. 3 and Table 3), whereas no detectable termination was observed for T $8 \Delta$. The wild-type thra terminator, that acts as an internal control, functioned normally (Fig. 3). Therefore, changes of deoxynucleotides downstream from the transcription termination site were probably responsible for the decrease in the termination efficiency between T8 $\Delta$ and T8WT. Such changes can also explain why the chloroplast enzyme failed to terminate at Tvar in the nested deletion variants which lacked the 5-nt downstream sequence (Fig. 2, lanes T8-T1).

In Figure 3, the transcripts that terminate at the wild-type thra terminator (Twt) with $T 8 \Delta$ template migrated more rapidly and were more abundant than the transcript terminated at templates T9WT and T8WT. The smaller product could be due to the $5 \mathrm{nt}$ deleted in the Tvar region, and its greater abundance was likely due to the lack of any transcript termination at the Tvar terminator, causing more transcripts to terminate at Twt.

\section{Downstream sequence affects termination in a sequence-specific manner}

The $3^{\prime}$ ends of the transcripts produced by chloroplast RNA polymerase at the thra terminator corresponded to the 7th or 8th deoxyadenosines downstream with respect to the $d(G+C)$-rich region $(1,31)$. This site of termination was essentially the same as that produced by E.coli RNA polymerase. When the sequences downstream from termination site in the wild-type (T8WT) and the deletion variant (T8A) are compared, only 3 out of $5 \mathrm{nt}$ are different (CGACT versus CTAGA). Thus, it becomes of interest to investigate further how these downstream sequences affect the termination efficiency. To address this question, we introduced several mutations within these $5 \mathrm{nt}$ by PCR with a degenerate primer YNANN (Fig. ID, Y $=\mathrm{C}$ or $\mathrm{T}, \mathrm{N}=\mathrm{G}, \mathrm{A}, \mathrm{C}$ or T). DNA fragments amplified with PCR were isolated and cloned into the BamHI site of pTZ-PLSTa as described in Materials and Methods. More than 80 transformants that contained various sequences in this 5-nt region were identified and tested in vitro for transcription termination by chloroplast RNA polymerase.

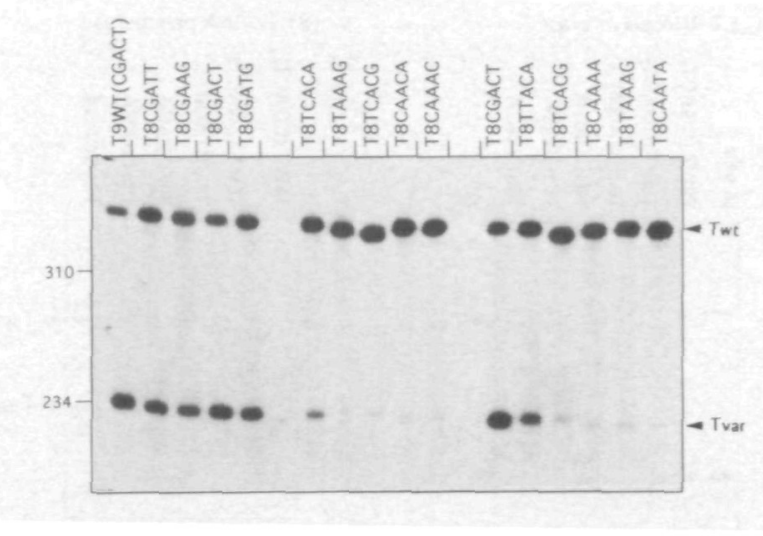

Figure 4. In vitro transcription of the thra terminator YNANN variants with chloroplast RNA polymerase. Transcription results of some of the YNANN variants are shown. The sequences composition for each YNANN variants is shown on top of each lane. Other designations, see the legend to Figure 3.

Examples of the transcription results are shown in Figure 4. These results indicate that when $\mathrm{G}$ appeared in the second nucleotide of this region, transcription terminated efficiently, whereas any other of the $3 \mathrm{nt}(\mathrm{A}, \mathrm{C}, \mathrm{T})$ in the second position failed to terminate (Fig. 4). A mutant that contained a 10-T tract (2 additional Ts) terminated to some extent, but less efficiently than CGNNNcontaining templates. In contrast, mutants having those variations in the 4th or 5th nucleotide contributed no significant effect on termination under the standard reaction conditions.

\section{Changing $T$ to $G$ in $T 8 \Delta$ restores the termination efficiency to near wild-type value}

To demonstrate further that the nucleotide located in the second position after the poly( $\mathrm{T})$ tract plays an important role in transcription termination, we constructed three variants designated T8 $\triangle$ CGAGA, T8 $\triangle$ CAAGA and T8 $\triangle \mathrm{CCAGA}$ in addition to the original variant, T8 $\triangle \mathrm{CTAGA}$ (Fig. 1D). Their sequences were confirmed to be identical except at the nucleotide underlined (Fig. 1D). These three variants along with T8 $\triangle \mathrm{CTAGA}$ (the same as $\mathrm{T} 8 \Delta$ ) were subjected to in vitro transcription by chloroplast RNA polymerase (Fig. 5A) or T7 RNA polymerase (Fig. 5B). The results (Fig. 5A) indicated that with chloroplast enzyme the variant T8 $\triangle$ CGAGA had a termination efficiency nearly the same as the wild-type construct, whereas the original template clone

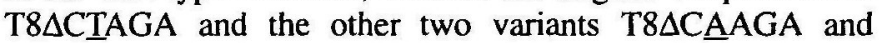
T8 $\triangle C C A G A$ had very inefficient or undetectable termination efficiency. These results clearly demonstrate that the $\mathrm{G}$ nucleotide in the second position of the downstream sequence plays an important role in termination efficiency. Whereas T7 RNA polymerase is not influenced by the specific nucleotide present at this position (Fig. 5B and Table 3).

\section{DISCUSSION}

The transcription termination model of the factor-independent terminator for E.coli RNA polymerase proposed by Yager and von Hippel $(6,7)$ required the $d(G+C)$-rich hairpin and the downstream poly $(T)$ tract for efficient termination $(6,8,30)$. Requirement of a stable $\mathrm{G}+\mathrm{C}$ hairrpin has been demonstrated for E.coli $(16,34)$ and $T 7(13,14)$ RNA polymerases with various 


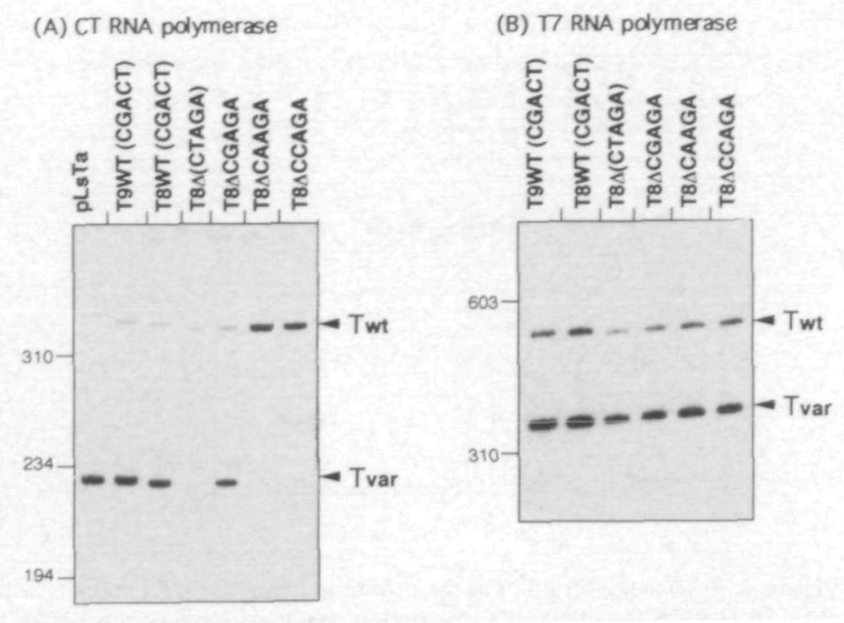

Figure 5. In vitro transcription of the thra terminator variants derived from T8 $\triangle$ CNAGA with chloroplast or T7 RNA polymerase. Transcription results of T8 $\triangle$ CTAGA, T8 $\triangle$ CGAGA, T8 $\triangle$ CAAGA, T8 $\triangle C C A G A$ and T8WT, T9WT with (A) chloroplast or (B) T7 RNA polymerase are shown. The specific nucleotide changed for each variants are shown on top of each lane. Other designations, see the legend to Figure 3 . For the experiment shown in (A), the template T8 $\triangle$ CTAGA gave less Twt transcript than usually observed (compare

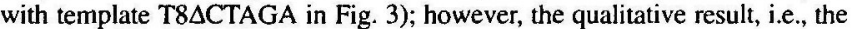
absence of Tvar transcript, is as before.

terminator mutants that destroy the helix integrity and destabilize the hairpin structure. However, the role of the instability of the dA-rU base pairs in the transcription complex has been brought into question by the findings that functioning $\mathrm{dA}-\mathrm{r} U$ runs may be as short as two base pairs (32) and that there are very strong terminators that lack the poly(T) tract (33).

In this study, using chloroplast RNA polymerase, we show that even though mutations that alter helix integrity and stability in the $\mathrm{G}+\mathrm{C}$-rich hairpin region can decrease termination efficiency, there is no consistent correlation between the termination efficiency and helix stability (Table 2). This finding is consistent with previous observations for termination by the chloroplast enzyme using different $E$.coli terminators having varying helix stabilities (1). Transcription by T7 RNA polymerase with the same thra terminator variants (13) used in this study and the in vivo study of the tR2 terminator of coliphage $\lambda$ (35) demonstrated that factors in addition to helix stability are necessary for efficient termination.

The lack of direct correlation between helix stability and termination obtained in this study implies that factors other than helix stability might be involved in termination efficiency. For example, variant $138 \mathrm{U}(-2 \mathrm{lkcal} / \mathrm{mol})$ converts the $\mathrm{C}$ at position 100 to $U$ to give a UG base pair and results in a termination efficiency value of $28 \%$, whereas variant $153 \mathrm{~A}(-16 \mathrm{kcal} / \mathrm{mol})$ changes this CG base pair to a CA mismatch and surprisingly has a $48 \%$ termination efficiency. Another example with a quite different termination efficiency occurred with variants $140 \mathrm{~A}$ $(26 \%)$ and $151 \mathrm{~A}(43 \%)$. Both have their sequences changed at the same base pair positions (position 102 and 113, respectively, Fig. $1 B)$ and have the same calculated helix stability $(-17 \mathrm{kcal} / \mathrm{mol})$. These observations vividly demonstrate that the sequence itself in the $\mathrm{G}+\mathrm{C}$-rich region can also influence the termination with chloroplast enzyme. However, the results obtained with chloroplast RNA polymerase are slightly different from those reported with T7 RNA polymerase (13 and Table 2). In that study, using T7 RNA polymerase, the termination efficiency of $138 \mathrm{U}$ is similar to that of 153A; likewise, the termination efficiency of $140 \mathrm{~A}$ is similar to that of $151 \mathrm{~A}$ (Table 2). Therefore, the differences in termination in these mutants can be attributed to differences between chloroplast RNA polymerase and T7 RNA polymerase.

In addition to the $\mathrm{G}+\mathrm{C}$-rich hairpin, the roles of sequence elements of the poly(T) stretch and poly(A) tract preceding the hairpin that might affect the termination efficiency of thra terminator have also been reported with E.coli $(16,34)$ or T7 $(13,14)$ RNA polymerases. In transcription by T7 RNA polymerase, mismatches in the $\mathrm{G}+\mathrm{C}$-rich region (variants $140 \mathrm{~A}$, $151 \mathrm{~A}, \mathrm{AD} 1, \mathrm{BC10}, 138 \mathrm{U}$ and $153 \mathrm{~A}$ ) resulted in lower termination efficiencies than observed in the variants $(160 \mathrm{~A}, 160 \mathrm{C}$, 160G, 1316UA and 1316GC), which contained a mismatch in the A+T-rich region (Table 2). In contrast, the results with the chloroplast enzyme reveal that three mismatch variants $140 \mathrm{~A}$, $151 \mathrm{~A}, 153 \mathrm{~A}$ and variant $138 \mathrm{U}$ in the $\mathrm{G}+\mathrm{C}$-rich region had termination efficiencies of $26-48 \%$, whereas the variants (160A, $160 \mathrm{C}, 160 \mathrm{G}, 1316 \mathrm{UA}$ and $1316 \mathrm{GC}$ ) that each has an altered nucleotide in the middle of $\mathrm{T}$ tract terminated transcription with efficiencies between 12 and $20 \%$ (Table 2). These observations indicate that, in general, mutations located at the $\mathrm{T}$ tract region affect termination efficiency by the chloroplast enzyme more than mutations located in the $\mathrm{G}+\mathrm{C}$-rich hairpin.

Studies with the nested deletion variants have been reported with E.coli (16) and T7 (13) RNA polymerases. The results indicated that with the $\mathrm{T} 7$ enzyme, templates with a $(\mathrm{dA})$ tract of four or fewer fail to terminate transcription, whereas templates with a deoxyadenosine tract of $\geq 5$ increase the termination efficiency from $30 \%$ (T5) to $90 \%$ (T8) of the wild-type value (13). Termination with the T8 construct is similar to that with the wild-type $\mathrm{T} 9$, suggesting that the sequences immediately downstream from the transcription termination site do not greatly affect termination with T7 RNA polymerase (13). This view is supported further by transcription with the T7 enzyme of variants

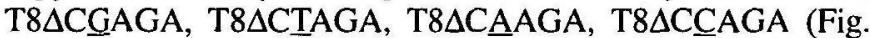
$5 B$ and Table 3); the YNANN mutants (data not shown); and the wild-type of T8WT and T9WT (Fig. 5B and Table 3). In contrast with E.coli or T7 RNA polymerase, the spinach chloroplast RNA polymerase had little or no termination with any of the shortened $\mathrm{T}$ tract variants (Fig. 2, lanes T8-T1). Sequences downstream from the termination release site, however, were crucial for termination efficiency. An observation with E.coli RNA polymerase also showed that the sequences between 3 and $7 \mathrm{nt}$ downstream of the T7Te terminator release site affected the strength of that terminator (17). However, the sequence requirement for transcription termination with chloroplast RNA polymerase is much more specific than that with E.coli RNA polymerase. Termination efficiency with the chloroplast enzyme was largely influenced by a single nucleotide at a defined position.

Several plausible mechanisms explain how sequences downstream from the release sites could affect termination efficiency. Transcriptional pausing during transcription may play a role in inducing termination, and several reports demonstrate that certain pause sites are encoded by sequences distal to the site of the pause $(36,37)$. Therefore, the specific downstream sequences that are responsible for the termination efficiencies in this study could be those that induce the RNA polymerase to pause and thus to enhance termination. Since transcriptional elongation requires 
opening of the DNA double helix, the downstream sequences might also function by affecting the ability of the two DNA strands to be separated during elongation (17).

Levin and Chamberlin (36) have reported that transcriptional pausing can be determined by efficiency with which polymerase utilizes its nucleotide substrates. The utilization of nucleotide substrates is sequence-dependent and the sequence-dependent $K s$ (dissociation constant) differences have been estimated to vary up to 500 -fold (36). The mechanism that determines the sequencedependent $K s$ differences for pausing is unknown; however, changing nucleotide concentrations in the transcription reaction affects the termination efficiencies of various terminators with E.coli RNA polymerase (33). Telesnitsky and Chamberlin (17) also reported that sequences downstream of T3Te affect termination efficiency dramatically as a function of the nucleotide concentrations. Therefore, it is possible that specific downstream sequences of the thra terminator affect the ability of chloroplast RNA polymerase to utilize substrates efficiently when it is paused at the hairpin, and consequently alter its termination efficiency.

Based on the model for transcription termination of Nudler $e t$ al. (27), the downstream sequences that are covered in the transcription complex may also function as inchworming signals to induce the termination possibly in conjunction with the poly $(T)$ tract. The specific downstream sequences that affect the termination of chloroplast RNA polymerase may also act as a site for binding to the RNA polymerase, providing the front-end signal that halts the $3^{\prime}$ edge of the transcription complex to facilitate inchworming and termination.

The chloroplast RNA polymerase used in this study is only partially purified and contains proteins other than those strictly required for transcription. Thus, it is possible that protein factors recognizing the downstream sequences might be involved in termination. Further investigation will be required to explore this possibility and the effects of nucleotide concentration variations.

In conclusion, this study demonstrates how changes in sequence and secondary structure can affect the way chloroplast RNA polymerase recognizes a classic tho-independent terminator. In Chlamydomonas chloroplast DNA, stem-loop structures can function as transcription terminators in vivo (4). This report demonstrates, by site-directed mutagensis, the importance of a stem-loop structure for transcription termination by a higher plant chloroplast RNA polymerase. In many cases the spinach enzyme responded like the E.coli and T7 RNA polymerases to variations in terminator sequence and secondary structure. However, in addition to helix stability, the specific sequence of the stem-loop was important for termination efficiency by the chloroplast RNA polymerase. Unexpectedly, we also observed that efficient termination by the spinach enzyme was critically dependent on sequence downstream from the thra termination site, in particular, on the presence of guanosine as the second nucleotide $3^{\prime}$ to the oligo-dT tract.

\section{ACKNOWLEDGEMENTS}

We thank Tim Setter, David Stern, Rita Monde and Chih-Ning Sun for discussions and critical reading of the manuscript. We thank $\mathrm{Li}$-Wen Fang for DNA sequencing. This work was supported by grants from National Science Council of the Republic of China, to L.-J.C.

\section{REFERENCES}

1 Chen, L.-J. and Orozco, E.M., Jr (1988) Nucleic Acids Res. 16, $8411-8431$.

2 Stem, D.B. and Gruissem, W. (1987) Cell 51, 1145-1157.

3 Stem, D.B., Jones, H. and Gruissem, W. (1989) J. Biol. Chem 264, 18742-18750.

4 Blowers, A.D., Klein, U., Ellmore, G.S. and Bogorad, L. (1993) Mol. Gen. Genet. 238, 339-349.

5 Chen, L.-J., Rogers, S.A., Bennet, D.C., Hu, M.-C. and Orozco, E.M., Jr (1990) Curr. Genet. 17, 55-64.

6 Yager, T.D. and von Hippel, P.H. (1987) Transcript elongation and termination in Escherichia coli in F.C. Neidhardt, S.L. Ingraham, K.B. Low, B. Magasanik, M. Schaechter and H.-E. Umbarger (eds) Escherichia coli and Salmonella typhimurium, Vol. 2, pp. 1241-1275, American Society for Microbiology, Washington, D.C.

7 Yager, T.D. and von Hippel, P.H. (1991) Biochemistry 30, 1097-1118.

8 Adhya, S. and Gottesman, M. (1978) Ann. Rev. Biachem. 47, 967-996.

9 Marin, F.H. and Tinoco, IJ. (1980) Nucleic Acids Res. 8, 2295-2299.

10 Farnham, PJ. and Plan, T. (1980) Cell 20, 739-748.

11 Famham, PJ. and Platt, T. (1981) Nucleic Acids Res. 9, 563-578.

12 Ryan, T. and Chamberlin, M.J. (1983) J. Biol. Chem. 258, 4690-4693.

13 Jeng, S.-T., Gardner, J.F. and Gumport R.I. (1990) J. Biol. Chem. 265, 3823-3830.

14 Jeng, S.-T., Gardner, J.F. and Gumport, R.I. (1992) J. Biol. Chem. 267, 19306-19312.

15 Lynn, S.P., Gardner, J.F. and Reznikoff, W.S. (1987) J. Bacteriol. 152. 363-371.

16 Lynn, S.P., Kasper, L.M. and Gardner, J.F. (1988) J. Biol. Chem. 263, $472-479$.

17 Telesnitsky, A. and Chamberlin, MJ. (1989) Biochemistry 28, 5210-5218.

18 Reynolds, B. and Chamberlin, MJ. (1992) J. MoL Biol. 224, 53-63.

19 Altmann, C.R., Solow-Cordero, D.E. and Chamberlin, M.J. (1994) Proc. Natl. Acad. Sci. USA, 91, 3784-3788.

20 Surrat, C.K. Milan, S.C. and Chamberlin, MJ. (1991) Proc. Natl. Acad. Sci. USA 88, 7983-7987.

21 Orlova, M., Newlands, J., Das, A., Goldfarb, A. and Borukhov, S. (1995) Proc. Natl. Acad. Sci. USA 92, 4596-4600.

22 Krummel, B. and Chamberlin, M.J. (1992) J. Mol. Biol. 225, 221-237.

23 Krummel, B. and Chamberlin. M.J. (1992) J. Mol. BioL 225, 239-250.

24 Nudler, E., Goldfarb, A. and Kashlev, M. (1994) Science 265, 793-796.

25 Chambertin, MJ. (1994) Harvey Lect. 88, 1-21.

26 Wang, D., Meier, T., Chan, C.L., Feng, G., Lee, D.N. and Landick, R. (1995) Cell 81, 341-350.

27 Nudler, E., Kashlev, M., Nikiforov, V. and Goldfarb, A. (1995) Cell 81, $351-357$.

28 Chen, L.-J. (1995) Bot. Bull. Acad. Sin 36, 95-100.

29 Freier, S.M., Kierzek, R., Jaeger, J.A., Sugimoto, N., Canuthers, M.H., Neilson, T. and Tumer, D.H. (1986) Proc. Nath. Acad. Sci. USA 83, 9373-9377.

30 Plath, T. (1986) Ann. Rev. Biachem. 55, 339-372.

31 Gardner, J.F. (1982) J. Biol. Chem. 257, 3896-3904.

32 Bean, B., Koren, R. and Mildvan. A. (1977) Biochemistry 16, 3322-3333.

33 Reynolds, R., Bermudz, R.M. and Chamberlin, MJ. (1992) J. MoL BioL $224,31-51$.

34 Yang, M.-T. and Gardner, J.F. (1989) J. Biol Chem. 264, 2634-2639.

35 Cheng, S.C., Lynch, E.C., Leason, K.R., Courn, D.L., Shapiro, B.A. and Friodman, D.I. (1991) Science 254, 1205-1207.

36 Levin, J.R. and Chamberlin, M.J. (1987) J. Mol. Biol. 196, 61-84.

37 Lee, D.N., Phung, L., Stewar, J. and Landick, R. (1990) J. Biol. Chem. 265, 15145-15153. 